\title{
Recent Evidence and Initial Experiences of Transcatheter Edge- to-Edge Repair of the Mitral Valve in South Korea
}

\author{
Sung-Jin Hong, M.D., Jung-Sun Kim, M.D., Ph.D., Geu-Ru Hong, M.D. \\ Division of Cardiology, Severance Cardiovascular Hospital, Yonsei University Health System, Seoul, Korea
}

\section{ARTICLE INFO}

Received January 28, 2021

Accepted February 9, 2021

Corresponding author

Jung-Sun Kim

Tel $82-2-2228-8457$

Fax 82-2-393-2041

E-mail kjs1218@yuhs.ac

ORCID

https://orcid.org/0000-0003-2263-3274

\begin{abstract}
As a percutaneous technique for the reduction of mitral regurgitation, the MitraClip system (Abbott Vascular, Abbott Park, IL, USA) for transcatheter edge-to-edge repair of the mitral valve was developed in 1998 and first used in 2003. Its main advantage is being less invasive than surgery, because it can be performed through a transcatheter approach without any hemodynamic compromise. Recent studies have shown that this procedure reduces symptoms and improves functional capacity with low complication rates. Two randomized clinical trials have investigated the use of this technique for functional mitral regurgitation. The Korean Ministry of Food and Drug Safety approved its use for degenerative mitral regurgitation in 2019, and this procedure started to be performed in Korea in January 2020. Its use for functional mitral regurgitation was also approved in Korea in 2020. In this article, recent evidence on transcatheter edge-to-edge repair of the mitral valve and our initial experiences in Korea will be reviewed.
\end{abstract}

Keywords: Mitral regurgitation, Transcatheter edge-to-edge repair, Heart valve disease, Mitral valve repair

\section{Introduction}

As a percutaneous technique to reduce mitral regurgitation (MR), the MitraClip system (Abbott Vascular, Abbott Park, IL, USA) for transcatheter edge-to-edge repair (TEER) of the mitral valve was developed in 1998 and first used in 2003. The main advantage of the TEER procedure that it is less invasive than surgery because it can be performed via the femoral venous approach without any hemodynamic compromise. Furthermore, unlike other percutaneous cardiovascular interventions such as coronary or aortic valve interventions, contrast medium is not required.

Based on recent results and evidence from randomized clinical trials, the use of MitraClip system was permitted in Europe (Conformité Européene, CE Mark) in 2008. In the United States, the Food and Drug Administration approved it in 2013 for degenerative MR, and in 2018 for functional MR. The Korean Ministry of Food and Drug Safety approved its use for degenerative MR in 2019, and this procedure started to be performed in Korea in January
2020. Its use for functional MR was also approved in Korea in 2020. In this article, recent evidence regarding TEER and our initial experiences in Korea will be reviewed.

\section{Recent clinical trials and evidence for TEER}

After the phase I feasibility study of the Endovascular Valve Edge-to-edge Repair Study (EVEREST) trial with 55 patients [1,2], the EVEREST II trial randomized 279 patients with $3+$ or $4+$ MR to receive TEER with the MitraClip or open mitral valve surgery in a 2:1 ratio [3]. Patients with both degenerative and functional MR were enrolled, and functional MR was present in $27 \%$ of patients [3]. In the surgery group, $86 \%$ of patients were treated with mitral valve repair and $14 \%$ had mitral valve replacement. The primary endpoint, a composite of freedom from death, surgery for mitral valve dysfunction, and $3+$ or $4+M R$ at 12 months, was achieved in $55 \%$ of patients in the percutaneous repair group and $73 \%$ of those in the surgical group $(p=0.007)$ [3]. This difference was mainly driven by the 
need for surgery for mitral valve dysfunction (20\% in the percutaneous repair group versus $2 \%$ in the surgical repair group, $\mathrm{p}<0.001)$. However, major adverse events within the first 30 days occurred more frequently in the surgery group, mainly driven by the need for blood transfusions ( $13 \%$ versus $45 \%, \mathrm{p}<0.001$ ). Thus, though percutaneous repair was less effective at reducing MR than surgery, TEER was superior in terms of safety and showed similar improvements in clinical outcomes [3]. According to the secondary analyses, percutaneous repair resulted in greater preservation of left ventricular (LV) function and a smaller decrease in LV dimensions compared with surgery. When the EVEREST II population was followed for 5 years, the primary endpoint still favored the surgical group $(64.3 \%$ in the surgical group and $44.2 \%$ in the percutaneous repair group) [4]. However, there was no significant difference in the rate of death (21\% versus $27 \%$ ), and freedom from mitral valve surgery or reoperation remained the same after percutaneous repair or surgery beyond 6 months after the procedure. Improvements in symptoms and LV dimensions were maintained at 5 years in both groups [4]. The EVEREST II trial became the landmark trial for commercial approval in the United States, particularly for degenerative MR.

Functional MR is frequently present in patients with advanced heart failure. Optimal medical and cardiac resynchronization therapy have been proposed as first-line strategies for functional MR. Surgical intervention may be considered in patients who are refractory to currently available medical or cardiac resynchronization therapy as a class IIb indication because the beneficial effect of mitral valve surgery has not been proven by prospective randomized clinical trials $[5,6]$. Meanwhile, transcatheter mitral intervention can be better option with a less invasive strategy in high-risk population with surgery and comparable strategy to mitral valve surgery. In 2018, two important randomized trials that included the patients with functional MR and heart failure, the MITRA-FR (Percutaneous Repair with the MitraClip Device for Severe Functional/ Secondary Mitral Regurgitation) trial and COAPT (Cardiovascular Outcomes Assessment of the MitraClip Percutaneous Therapy for Heart Failure Patients with Functional Mitral Regurgitation) trial, were published [7,8]. It is interesting to note that these 2 trials gave different results, as the MITRA-FR trial failed to prove superiority for the primary endpoint, but the COAPT trial proved it. The MITRA-FR study randomized 307 patients with symptomatic LV dysfunction and significant secondary MR to either medical therapy or medical therapy combined with the
MitraClip procedure, and $92 \%$ of patients achieved an MR grade of 2+ immediately after the procedure [7]. There was no difference in the primary outcome of all-cause death and unplanned re-hospitalization for heart failure at 1 year, which occurred in 55\% of the intervention group and 51\% of the control group (odds ratio, 1.16; $\mathrm{p}=0.53$ ) [7]. In the other COAPT trial, a total of 614 patients with symptomatic heart failure and moderate-to severe or severe secondary MR to medical therapy or medical therapy and MitraClip repair [8]. The primary outcome was the rate of hospitalization for heart failure within 24 months, which was $35.8 \%$ per patient-year in the device group as compared with $67.9 \%$ in the control group (hazard ratio, 0.53 ; $\mathrm{p}<0.001$ ) [8]. Moreover, the powered secondary endpoint of death from any cause within 24 months was significantly lower, occurring in $29.1 \%$ of the patients in the device group versus $46.1 \%$ in the control group (hazard ratio, 0.62 ; $\mathrm{p}<0.001)$. All prespecified secondary endpoints including quality of life and functional assessments significantly improved in the MitraClip arm $[9,10]$.

The following reasons have been proposed for these discordant results [11-13]. First, the definitions of severe MR were different between European and American guidelines. MR was more severe in the COAPT trial than in the MITRA-FR trial. Second, the LV end-diastolic volumes were smaller in the COAPT trial than in the MITRA-FR trial (101 versus $135 \mathrm{~mL} / \mathrm{m}^{2}$ ). Third, more aggressive guideline-directed medical therapy was overseen by the screening committee in the COAPT trial. Fourth, the number of patients receiving more than 1 clip was higher in the COAPT trial, possibly explaining the higher proportion of patients with moderate-to-severe or severe residual MR at 1 year in the MITRA-FR trial (17\% versus 5\% in COAPT). In light of the apparently discordant results from 2 recent randomized controlled trials of mitral valve repair, the concept of the characterization of MR as proportionate or disproportionate to LV size was proposed to differentiate patients who can benefit from the TEER procedure as part of optimal treatment for chronic heart failure and systolic dysfunction $[11,12]$. Patients with heart failure, an LV ejection fraction (LVEF) of 30\%, an LV end-diastolic volume of 220 to $250 \mathrm{~mL}$, and a regurgitation fraction of $50 \%$ would be expected to have an effective regurgitant orifice area of approximately equal to $0.3 \mathrm{~cm}^{2}$ independent of specific tethering abnormalities of the mitral valve leaflets [11]. The $\mathrm{MR}$ in these patients is proportionate to the degree of LV dilatation and can respond to drugs and devices that reduce LV end-diastolic volume. In contrast, patients with an effective regurgitant orifice area of 0.3 to $0.4 \mathrm{~cm}^{2}$, but with 
an LV end-diastolic volume of only 160 to $200 \mathrm{~mL}$, exhibit degrees of MR that are disproportionately higher than would be predicted by LV end-diastolic volume. Thus, these patients were proposed to preferentially benefit from interventions directed at the mitral valve [11-13].

\section{Recommendations and indications for TEER according to current guidelines}

For the assessment of the feasibility of TEER, a rigorous echocardiographic assessment and multidisciplinary team consensus is required [14]. The anatomically favorable features of TEER are non-commissural pathology (medial, middle, lateral segments), no or minimal calcification, mean trans-mitral gradient $<4 \mathrm{~mm}$, mitral valve area $\geq 4$ $\mathrm{cm}^{2}$, and a grasping zone length $>10 \mathrm{~mm}$ [14]. For primary $\mathrm{MR}$, flail width $<15 \mathrm{~mm}$, flail gap $<10 \mathrm{~mm}$, single segment pathology, and normal leaflet thickness are also favorable features. For secondary MR, coaptation depth $<11 \mathrm{~mm}$ and coaptation length (overlap length) $\geq 2 \mathrm{~mm}$ are favorable.

The 2020 American College of Cardiology/American
Heart Association guideline, for the patients with primary MR, recommended that TEER is reasonable in severely symptomatic patients (New York Heart Association [NYHA] class III or IV) with primary severe MR and high or prohibitive surgical risk if the mitral valve anatomy is favorable for the repair procedure and the patient's life expectancy is at least 1 year, as a class IIa recommendation [5]. It is notable that the previous 2017 European Society of Cardiology guideline recommended that TEER may be considered in patients with symptomatic severe primary MR who fulfil the echocardiographic criteria of eligibility and are judged inoperable or at high surgical risk by the heart team, avoiding futility, as a class IIb recommendation [6].

As for chronic secondary MR, TEER is reasonable (class IIa) in patients with appropriate anatomy as defined on transesophageal echocardiography and with an LVEF between $20 \%$ and $50 \%$, LV end-systolic diameter $\leq 70 \mathrm{~mm}$, and pulmonary artery systolic pressure $\leq 70 \mathrm{~mm} \mathrm{Hg}$, in patients with chronic severe secondary MR related to LV systolic dysfunction $(\mathrm{LVEF}<50 \%)$ who have persistent severe
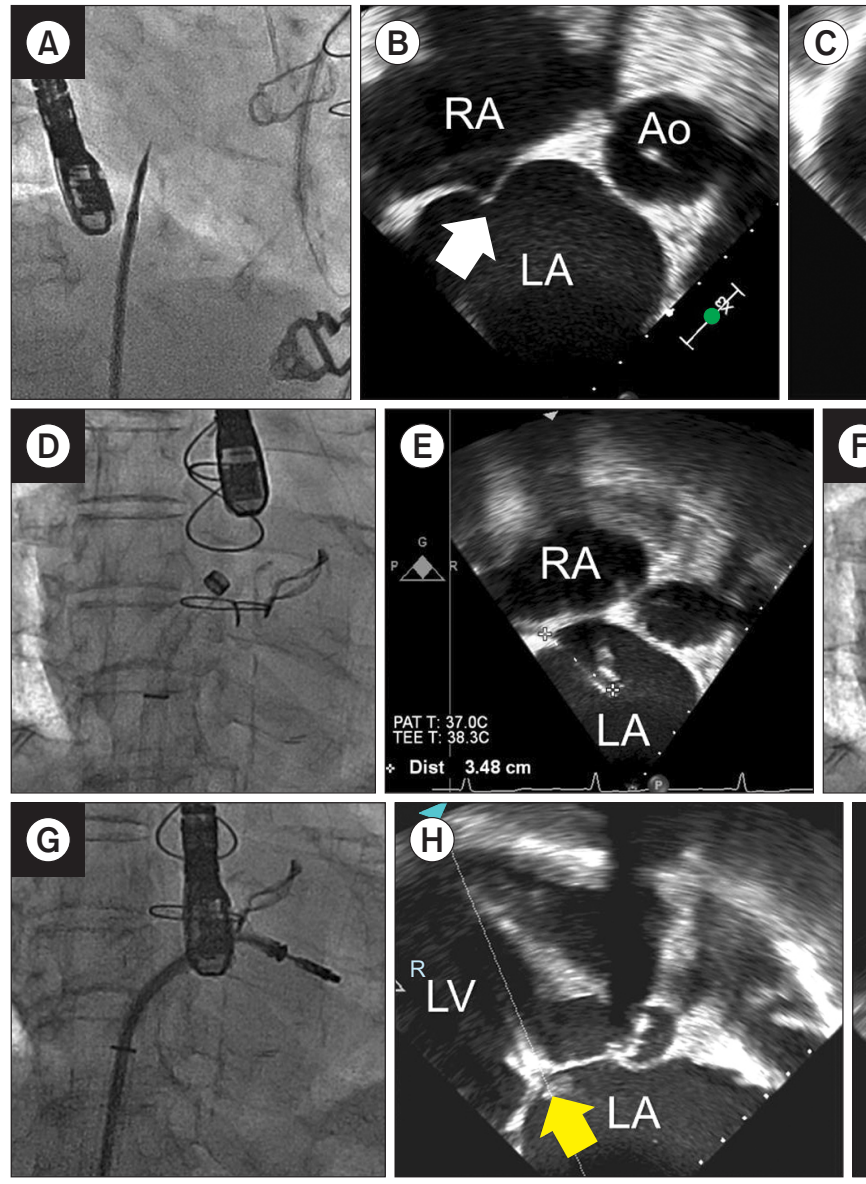

Fig. 1. Step-by-step procedure. (AC) Trans-septal pucture. Tenting of the atrial septum (white arrow) can be seen in transesophageal echocardiography (TEE) images of short-axis and 4-chamber views. (D, E) Introduction of a $24 \mathrm{~F}$ orientable guiding catheter to the left atrium (LA). (F) Introduction of a steerable clip delivery catheter. (G-I) Advance of the device into the left ventricle (LV) and grasping of the mitral valve (yellow arrow) under the left ventricular outflow tract view and intercommisural view on TEE images. RA, right atrium; Ao, aorta. 
symptoms (NYHA class II, III, or IV) while on optimal guideline-directed medical therapy for heart failure (stage D) [5]. Furthermore, we expect that the RESHAPE-HF2 (NCT02444338) and MATTERHORN (NCT02371512) trials might provide more concrete evidence and appropriate indications for the application of the MitraClip device for functional MR and the concept of disproportionate MR.

\section{Step-by-step procedure}

TEER is performed under general endotracheal anesthesia. To access the left atrium, a trans-septal puncture is performed via the femoral vein. One or two Perclose ProGlide devices (Abbott Vascular, Abbott Park, IL, USA) might be deployed in a pre-close fashion in the right common femoral vein followed by introduction of an $18 \mathrm{~F}$ sheath to help hemostasis after the procedure. Trans-septal puncture is performed in a posterior and mid-to-superior location on the interatrial septum using transesophageal echocardiographic guidance after confirmation of an adequate distance from the puncture location to the mitral annular plane (Fig. 1A-C). After trans-septal puncture, a $24 \mathrm{~F}$ orientable guiding catheter is percutaneously introduced (Fig. 1D, E), and a steerable clip delivery catheter enables orientation of the clip (Fig. 1F). Throughout the entire procedure, transesophageal echocardiographic guidance including real-time 3-dimensional images is important for the septal puncture, introduction of clip delivery system, positioning, and precision-targeting of the free edges of the opposing leaflets at the site of regurgitation. The device is then advanced into the left ventricle and while pulling back the catheter, the mitral valve leaflets are grasped (Fig. 1G-I).

Once optimal grasping has been undertaken, the clip is closed to create a double orifice (Fig. 2A, B). An echocardiographic assessment is performed for adequate leaflet insertion, residual MR with color Doppler (Fig. 2C, D), and new trans-valvular gradients avoiding mitral stenosis prior to the final deployment. Direct measurements of the left atrial mean pressure and $\mathrm{V}$ wave provide complementary hemodynamic data to guide treatment decision-making (Fig. 2E, F). Multiple clips can be implanted to optimize imperfect results on a case-by-case basis if the gradients and anatomy allow. Hemodynamics usually remain very stable during the procedure and the recovery time is short.
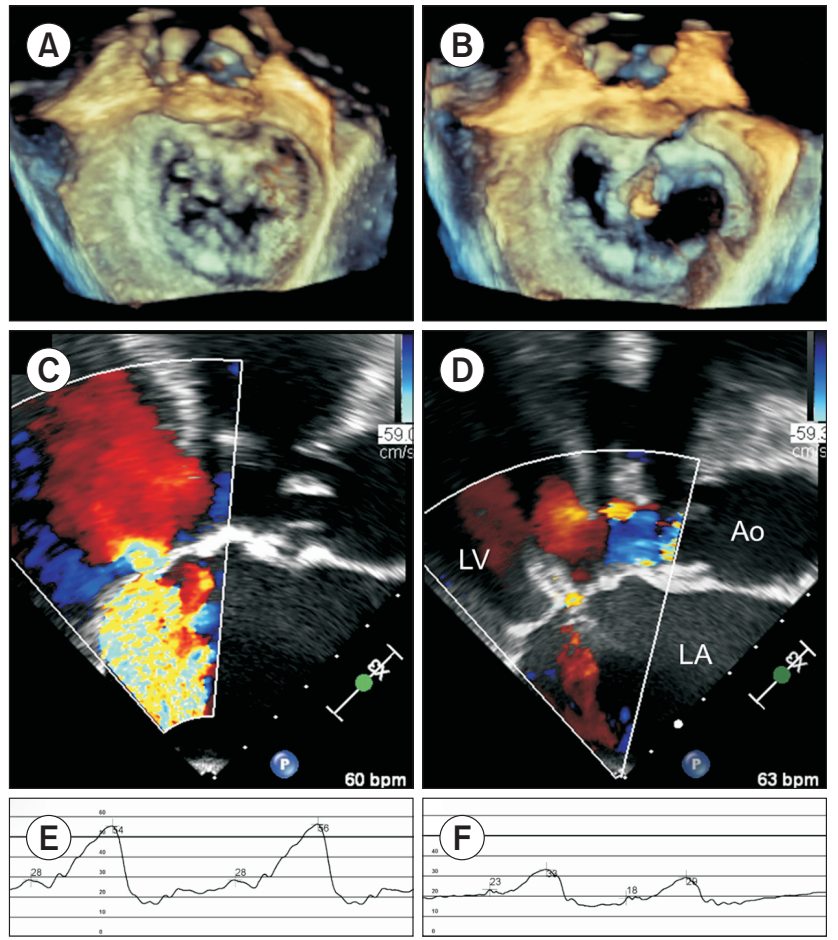

Fig. 2. Case example comparing before and after the procedure. (A) Before the procedure, A2 prolapse can be seen on 3-dimensional transesophageal echocardiography (TEE) images. (B) Creation of a double orifice after clip closure. (C) Severe mitral regurgitation (MR) on the left ventricular outflow tract view of the TEE image before the procedure. (D) After the clip closure, MR was reduced to a trivial degree. (E) Before the procedure, the mean left atrial (LA) pressure was $35 \mathrm{~mm} \mathrm{Hg}$ with a prominent $\mathrm{V}$ wave. $(\mathrm{F})$ After the procedure, the mean LA pressure was $21 \mathrm{~mm} \mathrm{Hg}$ with a decreased $V$ wave. Ao, aorta.

\section{Our initial experience and the results from major registries}

At Severance Cardiovascular Hospital, between February 2020 and January 2021, a total of 17 patients with moderate to severe or severe MR underwent TEER with the MitraClip NT system (Abbott Vascular), which is currently available in Korea. The patients' median age was 81 years (interquartile range, 76-86 years) and 10 patients (59\%) were women. The proportion of patients with NYHA III and IV functional status was 53\% and $47 \%$, respectively. The comorbidities were as follows: atrial fibrillation, 11 patients (65\%); hypertension, 15 patients (88\%); diabetes, 5 patients (29\%); and chronic kidney disease, 10 patients (59\%). At the time of the procedure, 1 patient (6\%) was in shock. The median EuroSCORE II was 5.0\% (interquartile range, $2.3 \%-6.9 \%)$. The median Society for Thoracic Surgeons (STS) scores for mitral valve repair and mitral valve 
replacement were $6.8 \%$ (interquartile range, $4.0 \%-9.1 \%$ ) and $9.0 \%$ (interquartile range, $8.0 \%-12.0 \%$ ), respectively. The overwhelming majority of patients $(88 \%)$ had STS scores $\geq 8 \%$ for mitral valve replacement. One patient had severe right ventricular dysfunction with severe tricuspid regurgitation. One patient had severe heart failure with chronic constrictive pericarditis.

Echocardiographic data are presented in Table 1. The median LVEF and end-diastolic diameter were $62 \%$ and 60

Table 1. Echocardiographic data $(n=17)$

\begin{tabular}{|c|c|}
\hline Variable & Value \\
\hline Left ventricular ejection fraction (\%) & $62(25-72)$ \\
\hline End-diastolic diameter (mm) & $60(46-66)$ \\
\hline End-systolic diameter (mm) & $40(31-49)$ \\
\hline$\geq 40 \mathrm{~mm}$ & $9(53)$ \\
\hline \multicolumn{2}{|l|}{ Mitral regurgitation severity } \\
\hline Grade 3 & $9(53)$ \\
\hline Grade 4 & $8(47)$ \\
\hline Effective regurgitant orifice area $\left(\mathrm{mm}^{2}\right)$ & $0.47(0.40-0.58)$ \\
\hline \multicolumn{2}{|l|}{ Etiology of mitral regurgitation } \\
\hline Degenerative & $12(71)$ \\
\hline Functional & $3(17)$ \\
\hline Degenerative and functional & $2(12)$ \\
\hline \multicolumn{2}{|l|}{ Tricuspid regurgitation } \\
\hline Grade 2 & $7(41)$ \\
\hline Grade 3 & $1(6)$ \\
\hline Grade 4 & $2(12)$ \\
\hline Right ventricular systolic pressure (mm Hg) & $48(23-62)$ \\
\hline \multicolumn{2}{|l|}{ Post-implantation mitral regurgitation } \\
\hline None/trivial & $2(12)$ \\
\hline Grade 1 & $5(29)$ \\
\hline Grade 2 & $9(53)$ \\
\hline Grade 3 & $1(6)$ \\
\hline Grade 4 & 0 \\
\hline Post-implantation mitral regurgitation grade $\leq 2$ & $16(94)$ \\
\hline Post-implantation mitral regurgitation grade $\leq 1$ & $7(41)$ \\
\hline
\end{tabular}

$\mathrm{mm}$, respectively. Before the procedure, 9 patients had grade $3+$ MR and 8 patients had grade $4+$ MR. Fig. 3A presents the procedural time (from the initiation of the trans-septal puncture to device deployment). It took a median of 129 minutes (interquartile range, 79-211 minutes; minimum, 70 minutes; maximum, 253 minutes). Twelve patients $(71 \%)$ had 1 clip implanted and 5 patients (29\%) required 2 clips; the average number of devices used was 1.29. Post-implantation MR severity is presented in Table 1, and post-implantation MR grade $\leq 2$ could be achieved in 16 patients (94\%) (Fig. 3B). The procedural complications and device-related adverse events were as follows: cardiac

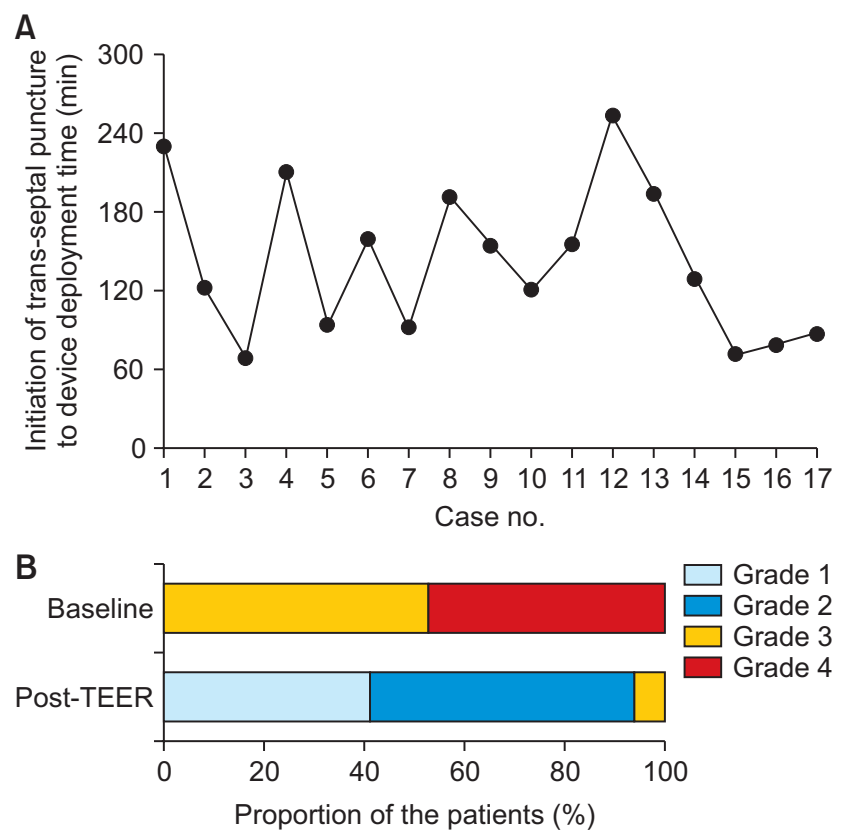

Fig. 3. Our initial experiences with the MitraClip (Abbott Vascular, Abbott Park, IL, USA). (A) Total time (minutes) between the initiation of trans-septal puncture to device deployment. (B) Severity of mitral regurgitation before and after the TEER procedure. TEER, transcatheter edge-to-edge repair.

Table 2. Summary of major registries of the transcatheter edge-to-edge repair procedure

\begin{tabular}{|c|c|c|c|c|c|c|c|c|c|}
\hline \multirow{2}{*}{ Variable } & \multirow{2}{*}{$\begin{array}{c}\text { No. of } \\
\text { patients }\end{array}$} & \multirow{2}{*}{ Age (yr) } & \multirow{2}{*}{$\begin{array}{c}\text { EuroSCORE } \\
(\%)\end{array}$} & \multirow{2}{*}{$\begin{array}{c}\text { STS } \\
\text { SCore }^{a)} \\
(\%)\end{array}$} & \multicolumn{2}{|c|}{ Etiology of MR (\%) } & \multirow{2}{*}{$\begin{array}{c}\text { Acute } \\
\text { procedural } \\
\text { success }(\%)\end{array}$} & \multirow{2}{*}{$\begin{array}{c}\text { 30-day } \\
\text { mortality } \\
(\%)\end{array}$} & \multirow{2}{*}{$\begin{array}{c}\text { 1-year } \\
\text { mortality } \\
(\%)\end{array}$} \\
\hline & & & & & Degenerative & Functional & & & \\
\hline ACCESS-EU [15] & 567 & $74 \pm 10$ & $22 \pm 18$ & - & 23 & 77 & 91 & 3.4 & 11.8 \\
\hline TCVT [16] & 628 & $74 \pm 10$ & $20 \pm 17$ & - & 28 & 72 & 95 & 2.9 & 15.3 \\
\hline TRAMI [17] & 749 & $76(71-81)$ & $20(12-31)$ & $6(4-11)$ & 28 & 71 & 97 & 4.7 & 20.3 \\
\hline STS/ACC TVT [18] & 2,952 & $82(74-86)$ & - & $9(6-14)$ & 86 & 9 & 92 & 5.8 & 25.8 \\
\hline Asia-Pacific Registry [19] & 142 & $71 \pm 12$ & $17 \pm 15^{b)}$ & $7 \pm 8$ & 53 & 47 & 94 & 5.6 & - \\
\hline
\end{tabular}

Values are presented as number, mean \pm standard deviation, or median (interquartile range).

MR, mitral regurgitation; TCVT, Transcatheter Valve Treatment Sentinel Pilot Registry; TRAMI, transcatheter mitral valve interventions; STS, Society of Thoracic Surgeons; ACC TVT, American College of Cardiology Transcatheter Valve Therapy.

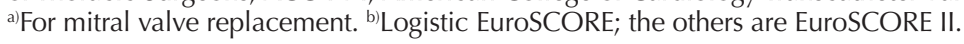


perforation $(\mathrm{n}=1)$, major bleeding requiring transfusion $(\mathrm{n}=1)$, mitral sub-valvular injury $(\mathrm{n}=1)$, transient ischemic attack $(\mathrm{n}=1)$, and air embolism $(\mathrm{n}=1)$. The 30 -day mortality rate was $6 \%(1 / 17)$. In-hospital death occurred in 2 patients. One patient successfully underwent TEER before liver transplantation, but liver failure progressed while waiting for a cadaveric donor, and he died 3 months after the procedure. One patient had heart failure with progressive constrictive pericarditis combined with severe MR. Although the reduction of MR was successful after the procedure, the patient died because of progression of heart failure and liver failure.

The results from the other major registries, including the Asia-Pacific Registry, are presented in Table 2 [15-19]. The mean ages were in the 70 s and 80 s, and the EuroSCORE was around $20 \%$. Acute procedural success was reported in $91 \%$ to $97 \%$ of cases, and the 30 -day mortality rate was $2.9 \%$ to $5.8 \%$

\section{Conclusion}

The TEER procedure has been introduced to reduce MR, and its main advantages are that it is less invasive than surgery, without any hemodynamic compromise or contrast use during the procedure. Though it started to be performed in Korea in January 2020 (i.e., later than in many other countries), our initial experience was acceptable compared to other recent registry data. For primary MR, TEER can be performed for patients with a high or prohibitive surgical risk if the mitral valve anatomy is favorable for the TEER procedure. For chronic secondary MR, patients with severe MR with symptomatic heart failure on optimal guideline-directed medical therapy can be considered for TEER. Furthermore, it is important to select patients through a multidisciplinary approach with heart team discussion.

\section{Conflict of interest}

No potential conflict of interest relevant to this article was reported.

\section{ORCID}

Sung-Jin Hong: https://orcid.org/0000-0003-4893-039X

Jung-Sun Kim: https://orcid.org/0000-0003-2263-3274

Geu-Ru Hong: https://orcid.org/0000-0003-4981-3304

\section{References}

1. Feldman T, Wasserman HS, Herrmann HC, et al. Percutaneous mitral valve repair using the edge-to-edge technique: six-month results of the EVEREST phase I clinical trial. J Am Coll Cardiol 2005;46: 2134-40.

2. Feldman T, Kar S, Rinaldi M, et al. Percutaneous mitral repair with the MitraClip system: safety and midterm durability in the initial EVEREST (Endovascular Valve Edge-to-Edge REpair Study) cohort. J Am Coll Cardiol 2009;54:686-94.

3. Feldman T, Foster E, Glower DD, et al. Percutaneous repair or surgery for mitral regurgitation. N Engl J Med 2011;364:1395-406.

4. Feldman T, Kar S, Elmariah S, et al. Randomized comparison of percutaneous repair and surgery for mitral regurgitation: 5-year results of EVEREST II. J Am Coll Cardiol 2015;66:2844-54.

5. Writing Committee Members, Otto CM, Nishimura RA, et al. 2020 ACC/AHA guideline for the management of patients with valvular heart disease: executive summary: a report of the American College of Cardiology/American Heart Association Joint Committee on Clinical Practice Guidelines. J Am Coll Cardiol 2021;77:450-500.

6. Baumgartner H, Falk V, Bax JJ, et al. 2017 ESC/EACTS guidelines for the management of valvular heart disease. Eur Heart J 2017;38: 2739-91.

7. Obadia JF, Messika-Zeitoun D, Leurent G, et al. Percutaneous repair or medical treatment for secondary mitral regurgitation. $\mathrm{N}$ Engl J Med 2018;379:2297-306.

8. Stone GW, Lindenfeld J, Abraham WT, et al. Transcatheter mitral-valve repair in patients with heart failure. N Engl J Med 2018; 379:2307-18.

9. Ailawadi G, Lim DS, Mack MJ, et al. One-year outcomes after MitraClip for functional mitral regurgitation. Circulation 2019;139:3747.

10. Arnold SV, Chinnakondepalli KM, Spertus JA, et al. Health status after transcatheter mitral-valve repair in heart failure and secondary mitral regurgitation: COAPT trial. J Am Coll Cardiol 2019;73:212332.

11. Grayburn PA, Sannino A, Packer M. Proportionate and disproportionate functional mitral regurgitation: a new conceptual framework that reconciles the results of the MITRA-FR and COAPT trials. JACC Cardiovasc Imaging 2019;12:353-62.

12. Packer M, Grayburn PA. New evidence supporting a novel conceptual framework for distinguishing proportionate and disproportionate functional mitral regurgitation. JAMA Cardiol 2020;5:469-75.

13. Yeo KK, Wong N. Percutaneous edge-to-edge mitral valve repair. Korean Circ J 2020;50:961-73.

14. Bonow RO, O'Gara PT, Adams DH, et al. 2020 Focused update of the 2017 ACC expert consensus decision pathway on the management of mitral regurgitation: a report of the American College of Cardiology Solution Set Oversight Committee. J Am Coll Cardiol 


\section{0;75:2236-70.}

15. Maisano F, Franzen O, Baldus S, et al. Percutaneous mitral valve interventions in the real world: early and 1-year results from the $A C$ CESS-EU, a prospective, multicenter, nonrandomized post-approval study of the MitraClip therapy in Europe. J Am Coll Cardiol 2013; 62:1052-61.

16. Nickenig G, Estevez-Loureiro R, Franzen O, et al. Percutaneous mitral valve edge-to-edge repair: in-hospital results and 1-year follow-up of 628 patients of the 2011-2012 Pilot European Sentinel Registry. J Am Coll Cardiol 2014;64:875-84.
17. Baldus S, Schillinger W, Franzen O, et al. MitraClip therapy in daily clinical practice: initial results from the German transcatheter mitral valve interventions (TRAMI) registry. Eur J Heart Fail 2012;14: 1050-5.

18. Sorajja P, Vemulapalli S, Feldman T, et al. Outcomes with transcatheter mitral valve repair in the United States: an STS/ACC TVT registry report. J Am Coll Cardiol 2017;70:2315-27.

19. Yeo KK, Yap J, Yamen E, et al. Percutaneous mitral valve repair with the MitraClip: early results from the MitraClip Asia-Pacific Registry (MARS). EuroIntervention 2014;10:620-5. 